\title{
Response of an electron system to a periodic potential
}

\author{
S. Nagy, K. Sailer \\ Department of Theoretical Physics, Debrecen University, Debrecen, Hungary
}

(October 31, 2018)

\begin{abstract}
We give a quantum field theoretical treatment of a one dimensional electron system with a fixed chemical potential $\mu$. The non-perturbative Lindhard response function is found for an electron system in a sinusoidal potential. 12.20.Ds
\end{abstract}

Typeset using REVTEX 


\section{INTRODUCTION}

Our goal is to treat relativistically the response of an electron system to a periodic electric field in the framework of $1+1$ dimensional $\mathrm{QED}\left(\mathrm{QED}_{2}\right)$. We assume the presence of a classical periodic electric mean field, i.e. the electromagnetic field variable $A_{\nu}(x)=\bar{A}_{\nu}+\alpha_{\nu}$ is the sum of this classical field and the quantum fluctuations. The interaction of the fermions with this classical field is treated exactly, whereas the quantum fluctuations of the

electromagnetic field are taken into account at 1-loop order. Requiring that the classical field is a mean field enables us to investigate a Wigner crystal (Wigner 1934, Grimes 1979) relativistically. To get a better understanding of such an electron system, it is unavoidable to investigate its non-perturbative response to a classical periodic electric field, that is the issue of the present work.

The classical periodic electric field is chosen in the form:

$$
\bar{A}^{\nu}(x)=a \delta_{0}^{\nu} \cos (\ell x)+\delta_{0}^{\nu} \mu
$$

with amplitude $a$ and wavenumber $\ell$. The homogeneous term $\mu$ corresponds to the chemical potential of the system.

\section{METHOD}

In QED the expectation value of the gauge field vanishes. To take into account a mean field self-consistently in this theory we should modify its generating functional.

Our method is based on defining a sector of QED for field configurations belonging to quantum fluctuations around a given vacuum expectation value $\left\langle A^{\mu}(x)\right\rangle$. We multipy the integrand of the common generating functional of $\mathrm{QED}_{2}$ by the factor 1 written in the form

$$
1=\int d c \delta\left(\int d x A_{\mu} n^{\mu}-c \Omega\right)
$$

where $n^{\mu}(x)$ is a vector in the space of the vector potential configurations and with $\Omega=T V$ the spacetime volume. Then, we find 


$$
Z_{Q E D}=\int d c Z_{Q E D}^{\prime}[n, c]
$$

where the functional

$$
Z_{Q E D}^{\prime}[n, c]=\int \mathcal{D} \bar{\psi} \mathcal{D} \psi \mathcal{D} A \exp \left\{\mathrm{i} S_{e m}[A, \xi]+\mathrm{i} S_{D}[A, \bar{\psi}, \psi]\right\} \delta\left(\int d x A_{\mu} n^{\mu}-c \Omega\right)
$$

is the generating functional of the sector belonging to vector potential configurations in a hypersurface orthogonal to $n^{\mu}(x)$. $A^{\mu}(x)$ is the electromagnetic and $\psi(x)$ is the Dirac field, $S_{\text {em }}[A, \xi]$ and $S_{D}[A, \bar{\psi}, \psi]$ are the action of the electromagnetic field and the Dirac action, resp., and $\xi$ is the gauge fixing parameter. By shifting the integration variable according to $\alpha^{\mu}(x)=A^{\mu}(x)-\bar{A}^{\mu}(x)$, the projected generating functional becomes

$$
\begin{aligned}
Z_{Q E D}^{\prime}[\bar{A}, c]= & \int d \lambda \int \mathcal{D} \alpha \exp \left\{\mathrm{i} S_{e m}[\bar{A}+\alpha, \xi]\right\} \exp \left\{\mathrm{i} \lambda\left(\int d x \bar{A}^{\mu} \bar{A}_{\mu}+\int d x \bar{A}^{\mu} \alpha_{\mu}-c \Omega\right)\right\} \times \\
& \int \mathcal{D} \bar{\psi} \mathcal{D} \psi \exp \left\{\mathrm{i} S_{D}[\bar{A}+\alpha, \bar{\psi}, \psi]\right\} .
\end{aligned}
$$

Self-consistency requires that

$$
\left\langle A^{\mu}(x)\right\rangle=\bar{A}^{\mu}(x), \quad \text { i.e. } \quad\left\langle\alpha^{\mu}\right\rangle=0 .
$$

This condition fixes the value of $c=\int d x \bar{A}_{\mu} \bar{A}^{\mu}$. To fulfill the requirement of (6) an extra renormalization condition is needed to every single loop correction. In our treatment the form of $\bar{A}_{\nu}$ coincides with (四). Calculations of any physical quantity is performed by taking an expectation value of that quantity with the modified generating functional.

To determine the Lindhard function we should calculate the polarization $\Pi\left(x, x^{\prime}\right)$ the form of which in QED is:

$$
\Pi_{\mu \nu}\left(x, x^{\prime}\right)=\frac{e^{2}}{i} \operatorname{tr}\left(\gamma_{\mu} G\left(x, x^{\prime}\right) \gamma_{\nu} G\left(x^{\prime}, x\right)\right),
$$

where $G\left(x, x^{\prime}\right)$ is the electron propagator.

Diagrammatically $\Pi\left(x, x^{\prime}\right)$ corresponds to the diagram in FIG. 1. (without the external legs). In FIG. 1. the solid lines represent the electron propagator and the wavy lines correspond to the photon propagator. Since we are interested first in the response of the electron system to an arbitrary periodic potential, $a$ and $\ell$ should be considered as free parameters in the Lindhard function. 


\section{RESULTS}

The electron propagator belonging to the projected generating functional contains all the effects of the periodic classical field and the propagator itself looses its translational symmetry due to this sinusoidal vector potential. Another consequence of the periodic classical field is that the electron propagator from (5) does not have analytic form, the Linhard response function can only be obtained numerically. First, we write down $\Pi_{\mu \nu}$ in momentum space according to:

$$
\Pi_{\mu \nu}\left(k, k^{\prime}\right)=\frac{e^{2}}{i} \int d x \int d x^{\prime} e^{i k x+i k^{\prime} x^{\prime}} \operatorname{tr}\left(\gamma_{\mu} G\left(x, x^{\prime}\right) \gamma_{\nu} G\left(x^{\prime}, x\right)\right) .
$$

In momentum space the two-vector $k=\left(k_{o}, \vec{k}\right)$ is the momentum of the photon in FIG. 1. coming from the left, while $k^{\prime}=\left(k_{o}^{\prime}, \vec{k}^{\prime}\right)$ denotes the momentum of the outgoing photon. Performing the integrations in (8) we get:

$$
\begin{aligned}
\Pi_{\mu \nu}\left(\vec{k}, k_{0} ; \vec{k}^{\prime}, k_{0}^{\prime}=-k_{0}\right)= & -e^{2} \sum_{k_{1} s_{1} k_{2} s_{2}} \sum_{n_{1} n_{2} n_{3} n_{4}} \frac{1}{\epsilon_{k_{1} s_{1}}^{(-)}+\epsilon_{k_{2} s_{2}}^{(+)}-2 k_{0}} \times \\
& \delta\left(\vec{k}+\vec{k}^{\prime}+\vec{\ell}\left(n_{3}+n_{4}-n_{1}-n_{2}\right)\right) \times \\
& \left(\mathcal{J}_{\mu \nu} \delta\left(\vec{k}_{1}+\vec{k}_{2}-\vec{k}+\vec{\ell}\left(n_{1}+n_{2}\right)\right)+\right. \\
& \left.\mathcal{J}_{\nu \mu} \delta\left(\vec{k}_{1}+\vec{k}_{2}-\vec{k}^{\prime}+\vec{\ell}\left(n_{1}+n_{2}\right)\right)\right),
\end{aligned}
$$

where $\epsilon_{k_{1} s_{1}}^{(-)}$and $\epsilon_{k_{2} s_{2}}^{(+)}$are positive energy eigenvalues corresponding to the positive and negative frequency eigenspinors of the Dirac Hamiltonian $H_{D}[\bar{A}]$ with the potential (1)), and $\mathcal{J}_{\mu \nu}$ contains products of the eigenspinors of $H_{D}[\bar{A}]$ in the following form:

$$
\mathcal{J}_{\mu \nu}=\bar{v}_{n_{2}}^{k_{2} s_{2}} \gamma_{\mu} u_{n_{1}}^{k_{1} s_{1}} \cdot \bar{u}_{n_{3}}^{k_{1} s_{1}} \gamma_{\nu} u_{n_{4}}^{k_{2} s_{2}}
$$

$u_{n}^{k s}$ and $v_{n}^{k s}$ are parts of the Bloch type eigenspinors (Nagy 2000). The first Dirac delta in (9) expresses the breaking of the translational symmetry due to the periodic field. We expect that (9) at close to $k_{0}=k_{0}^{\prime}=0$ has a maximum value for $\vec{k}=\vec{\ell}$ when the Fermi surface is positioned close to the boundary of the Brillouin zone. It would mean that there is no energy exchange between the two electrons in FIG. 1. but they exchange momentum $\vec{\ell}$. It 
reminds us to the so called Peierls phase transition where near the Fermi surface electrons can jump from one side of the Fermi surface to the other without any energy cost. The instability caused by this procedure is stabilized by a gap in the dispersion relation arising at the edge of the Fermi surface. In our treatment the stability is ensured by the periodic electric mean field which produces a gap at the edge of the Brillouin zone.

\section{ACKNOWLEDGEMENTS}

The authors would like to thank J. Polonyi and G. Plunien for the useful discussions. This work was supported by the projects OTKA T023844/97, DAAD-MÖB 27/1999 and NATO SA( PST.CLG 975722)5066. Numerical computations are in progress on the computer cluster donated by the Alexander von Humboldt Foundation.

\section{REFENCES}

Grimes, C. C. and Adams, G., Phys. Rev. Lett. 42, 795 (1979)

Nagy, S., Sailer, K., Heavy Ion Phys. 11, Nos 1-2, 67 (2000)

Wigner, E. P., Phys. Rev. 46, 1002 (1934)

\section{FIGURE CAPTIONS}

FIG. 1.: Diagram of $\Pi_{\mu \nu}\left(x, x^{\prime}\right)$. 


$$
-0
$$

